\title{
Connecting Mobile Users Through Mobile Social Networks
}

\author{
http://dx.doi.org/10.3991/ijim.v6i4.2224 \\ Faisal Alkhateeb, Ahmed M. Manasrah, Iyad Abu Doush and Eslam Al Maghayreh \\ Yarmouk University, Irbid, Jordan
}

\begin{abstract}
Nowadays, social networks become popular with the emerging of web-based social networking services. Recently, several mobile services are developed to connect users to their favourite social networks such as Facebook, Twitter, Flickr, etc. However, these services depend upon the existing web-based social networks. In this paper, we present a mobile service for joining groups across communities. The originality of the work is that the framework of the service allows creating and joining social networks that are self-contained for mobile company servers. The service consists of several sub-services such as users invitation, group finding and others. Users, regardless of their disability, can use the service and its sub-services without the need to create their own accounts on social web sites and thus their own groups. We also propose a privacy control policy for mobile social networks.
\end{abstract}

Index Terms-Social networks, mobile social networks, RDF.

\section{INTRODUCTION}

A social network is a social structure that may consists of individuals (or organizations) called "nodes", which are connected by one or more specific types of relationships, such as friendship, kinship, common interest, financial exchange, affiliation, etc [29]. The "social network" concept has been used several decades ago [25]. But, nowadays, social networks and social networking services become more popular with the contribution of the current facilities such as the web and mobile communications.

A social networking service is usually an online service (could be a platform or a site) [5] that aim at constructing social networks or social relations among people.

A social network service essentially consists of a representation of each user (often a profile), his/her social links, and a variety of additional sub-services [Site 1]. Most social network services are web based and provide means for their users to interact among themselves over the Internet, such as e-mail and instant messaging. Online community services are sometimes considered as a social network service, though in a broader sense, social network service usually means an individual-centered service whereas online community services are group-centered. Social networking sites allow users to share ideas, activities, events, and interests within their individual networks [14].

Recently, the number of social networks services has been increased and many of them are web-based such as Facebook [Site 2], Twitter [Site 3], LinkedIn [Site 4], MySpace [Site 5], Flickr [Site 6], Hi5 [Site 7], and others.
As the number of mobile phones users is on the rise, researchers and industry directed towards mobile social networking ${ }^{1}$.

Recently, several mobile social networking services have been developed such as Meet Now Live, sniff, Trapster,

Whrrl, Xumii, etc (refer to the related work section for more details). Nonetheless, these services depend upon the existing web-based social networking services. That is, the users can use these services to connect to social networks and keep in touch with their friends on Flickr, Facebook, MySpace, and others. Moreover, the users of mobile phones form a natural social network and, to the best of our knowledge, none of the existing mobile networking services benefit from this fact and thus building this social network.

In other words, most of the social networking are online services that emphasize on building and reflecting social relations among people, whereby users can share data/activities with others whom supposed to be their friends or friends of a friend. Most social network services are web-based and provide means for users to interact over the Internet, such as e-mail and instant messaging. However, computers are not always accessible, where mobile phones are. Therefore, a trend to turn the mobile phone into a social network is starting to arise. Especially, mobile phones provide services, such as phone calls and text messaging that are widely used. These services were designed for interpersonal communication and suitable for small groups of people, but they do not fit well for the coordination of larger groups [18], [2], [20]. Conversely, the advanced capabilities of mobile devices extend the scope of social interactions, beyond the small social group where people can now share their current location, phonebook contacts, calendar events and personal notes, and personal media (photos, video clips, etc) among others within the social group. However, such feature or flexibility imposes and raises higher awareness on privacy associated with shared information as well as the boundaries of the comfort level on what type of information can be disclosed and to whom and when. On the other hand, social websites provide various methods to users in order to easily publish their personal information on the Web. Yet, once published, users have no control on how their data should be accessed (i.e. who should access them and when/how?). In other words, most Social Networks provide a coarser-grained privacy settings restricting access to private data to those who are in the user's friends lists or user's groups such as Facebook's privacy preferences

\footnotetext{
${ }^{1} 800$ million users by 2012 of mobile social networking and $\$ 60$ billion
} in revenues [Site 8]. 
and Google $+[$ Site 15] circles. Users however, need to restrict their profile access to their friends list only after they are invited. Otherwise, open/public profile (i.e unrestricted) can be accessed by anyone even though requesters are not in the user friend list yet. As a result, users still demanding more complex privacy settings as discussed in [6].

In our side, we have presented a mobile social networking service that will be used for building a social network for mobile phone users and their connections [31]. The proposed service consists of several sub-services that will be discussed in details in the paper. Our work is motivated by the fact that the number of mobile users is more than the number of Web users and users can use the service and its subservices without the need to create their own accounts on social web sites and thus their own groups. In this paper, we provide more details about the service and show how users regardless of their disabilities, for example deaf or blind persons, can benefit from the proposed service. In addition, we extend our work [31] by proposing fine-grain privacy policy for restricting or granting access to specific data on mobile social networks.

Paper Outline. The remainder of the paper is organized as follows. In Section II, we introduce RDF and the SPARQL language. Section III is dedicated to the presentation of the proposed service. We propose in Section IV a privacy control policy for the mobile social networks. We discuss other related work in Section V. Finally, we conclude our work in Section VI.

\section{PRELIMINARIES}

This section provides an overview of the elements that are necessary for presenting the proposed approach namely: RDF and SPARQL. RDF is basically a graphbased language, which makes it very suitable for representing social networks (as they have a graph nature) while SPARQL will be used for searching RDF data.

\section{A. $R D F$}

RDF (Resource Description Framework) [21] is a knowledge representation language dedicated to the annotation of resources within the Semantic Web. Currently, many documents are annotated via RDF due to its simple data model and its formal semantics. In its abstract syntax, an RDF document is a set of triples of the form 〈subject, predicate, object〉.

Example 1: The assertion of the following RDF triples:

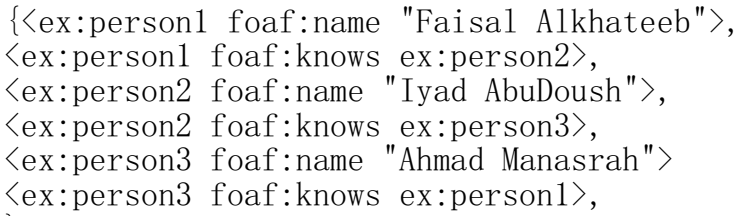

means that there exists a person named "Faisal Alkhateeb" who knows another person named "Iyad AbuDoush" who in turn knows a third person named "Ahmad Manasrah". Note that foaf [Site 9] and vcard [Site 10] (which is used in this paper), are two RDF vocabularies.

An RDF document can be represented by a directed labeled graph, as shown in Figure 1, where the set of nodes is the set of terms appearing as a subject or object in a triple and the set of arcs is the set of triples (i.e., if $<s, p$, $\mathrm{o}>$ is a triple, then $\mathrm{s} \rightarrow \mathrm{o}$ is an arc labeled with $\mathrm{p}$ ).

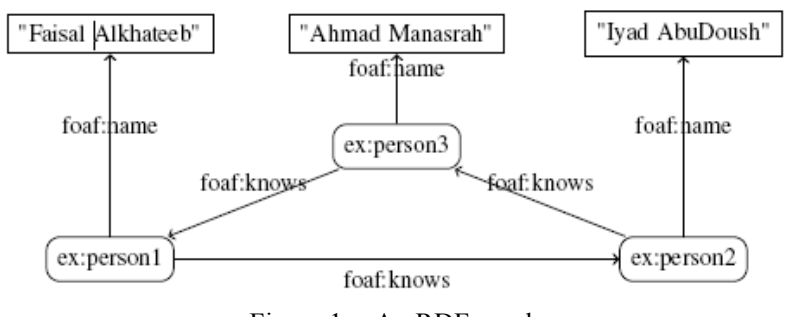

Figure 1. An RDF graph.

\section{B. SPARQL}

SPARQL is a $\mathrm{W} 3 \mathrm{C}$ recommendation language developed in order to query RDF knowledge bases, e.g. retrieving nodes from RDF graphs [24]. A simple SPARQL query is expressed using a form resembling the SQL SELECT query:

\section{SELECT B FROM $\mathrm{u}$ WHERE P}

where $\mathrm{u}$ is the URL of an RDF graph $\mathrm{G}$ to be queried, $\mathrm{P}$ is a SPARQL graph pattern (i.e., a pattern constructed over RDF graphs with variables) and B is a tuple of variables appearing in P. Intuitively, an answer to a SPARQL query is an instantiation of the variables of $\mathrm{B}$ by the terms of the RDF graph $G$ such that the substitution of the values to the variables of $\mathrm{P}$ yields to a subset of the graph $\mathrm{G}^{2}$.

Example 2: The following SPARQL query:

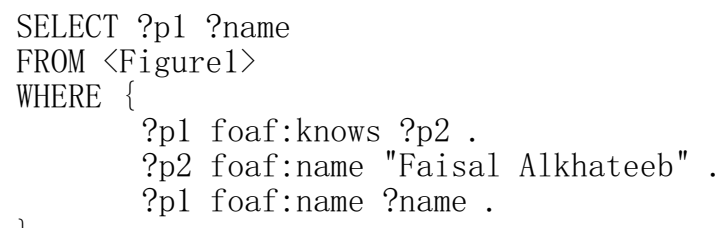

could be used to retrieve the name of persons who know a person named "Faisal Alkhateeb". When evaluated against the RDF graph of Figure 1, the following answers will be returned:

\begin{tabular}{|c|c|c|}
\hline$\#$ & $? p 1$ & ?name \\
\hline 1 & ex: person1 & "Ahmad Manasrah" \\
\hline
\end{tabular}

\section{THE PROPOSED SYSTEM}

\section{A. Design}

One of the goals of the proposed service is to build a social network for mobile users. The social network will be created incrementally based upon the mobile users profiles as well as their call and message connections. To this extent, we use the RDF language for representing users' profiles and so building the social network, i.e.,

\footnotetext{
${ }^{2}$ When using RDFS semantics [8], this intuitive definition is irrelevant and one could apply RDFS reasoning rules to calculate answers over RDFS documents.
} 
PAPER

each user profile will be represented as an RDF knowledge base (see Figure 2). In this RDF knowledge base, the username is used for identifying (and thus searching) user profiles since it is a unique key. The obtained RDF knowledge base will be maintained in the server of the mobile company.

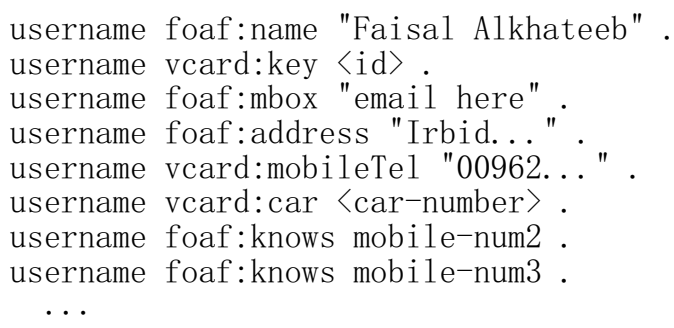

Figure 2. An RDF graph representing a part of a user profile.

Building the social network of mobile users in the server of the mobile company has several benefits. These benefits are described next:

\section{1) Profile activation}

Any user that wants use the service and its sub-services should activate his/her profile. This is achieved through an SMS sent by the mobile company to the user in order to fill his/her information (such as his name, international identification number ${ }^{3}$, address, car number if any, etc). The company will then provide the user with his/her username that could be a combination of his/her name and/or id.

\section{2) Profile updating}

After profile activation, the user can use his usernmae for updating his profile. In this case, the mobile company will notify his/her group members with any changes to his/her mobile number.

\section{3) Users invitation}

Any user $\mathrm{x}$ can send an SMS message to another user $\mathrm{y}$ to invite him/her to his/her group. Additionally, users' invitation service could be automatically invoked based upon some heuristics such as the number of call connections between both users and intersection between their profiles. If $y$ accepts the invitation then an RDF triple is added to their both profiles (to the RDF knowledge base) using the foaf:knows property and their identification number ${ }^{4}$. That is, if mobile-numX (respectively, mobilenumY) is the identification URL of user $x$ (respectively, user y), then the RDF triple <mobile-numX foaf: knows mobile-numY> (respectively, $\langle$ mobile-numY foaf:knows mobile-numX>) is added to the profile of $\mathrm{x}$ (respectively, profile of $y$ ).

\section{4) Instant messaging}

This service can be used by a given user to send a message to the group members with whom he has connection.

\section{5) Group finding}

A mobile social network system enables people to be engaged into friendship using the mobile phone [17]. The

\footnotetext{
${ }^{3}$ The international identification number (iin) is mandatory. For those countries that do not use iin, the international mobile phone number could be used.

${ }^{4}$ We can use PSPARQL [3], our extension to SPARQL, to find friend of friend.
}

cities are expanding and changing continuously, and the people are on the move most of the time [11]. Location based services (LBS) can provide the people with services with regard to their location. The geographical position of the person is identified by matching the mobile terminal with the mobile network. The LBS can be used for several purposes: healthcare, friends making, or finding the nearest store. Hewett et al. [1] indicates that blind and visually impaired people use social networking sites mostly to communicate through chat functions and to stay connected with friends. The mobile-based social network can provide several services to people with disabilities. With the addition of the services identified in the previous section it can provide the following services:

- Identify the location of a person or a place by using their profile and locating their geographical position using LBS. For example, a blind person wants to meet his friend who is in another building in a university. The LBS will identify the position of the blind person and the position of his friend using LBS. Then these positions will be passed to the maps in the phone to help the blind person in the navigation.

- Looking for people with specific characteristics or interests. For example, a deaf person can look for all deaf people in the surrounding area who identify their profiles as public. Another example is a blind person who is interested to see if any of his friends is going to the festival tonight. The user can use this sub-service to locate the group members based on the constructed social network with the following two options: WI-FI or zone limit:

O If the user selects the WI-FI limit option, then the service provides the user with the group members that are located around him/her via WI-FI distance limit upon request. The user can then selects one or more of the located group members and can send to them files, perform instant chatting or can call of one of them for urgent purpose. It is possible to use bluetooth limit instead of the WI-FI limit. In this case, an application should be deployed in the user mobile. The application interacts with the bluetooth to retrieve mobile names of group members located around him/her via WI-FI distance limit upon user request. Then it interacts with the service so that the mobiles names retrieved by the bluetooth are refined and are restricted to only mobiles names of the group members. Also, this option requires that the mobile name of each group member is the same as the member identification name (i.e., A unique username is provided by the company to the mobile user and it is a combination of his/her name and the mobile number).

- If the user selects zone limit option, then the service will provide the user with the group members located in the user zone. Note that the zones are defined by the mobile phone company. For example, Yarmouk University zone is the zone reserved for Yarmouk University. This service invokes the tracking location-based service [27], [26], [4], [10], [28] to locate each group member of a given user. The name and the mobile number is provided to the user if the group member is located in the same zone of the user invoking this service. Moreover, a group member should provide the 
PAPER

permission to the service to know his/her location by any of other group members.

\section{6) Profile finding}

This service can be used when a given user has a problem with a given mobile or a car. In this case, he/she can use this service to retrieve the knowledge (profile) about the owner of that car or that mobile number as well as the group knowing him/her. This is achieved based upon the car number or the mobile number as a key for searching. The user can then call him/her or one of the retrieved group members. This is important since it often happened that one car hits another or one person cannot exit his/her car because another car closed the road. In more details, if the user selects this service then he/she has several search options:

- Searching based on a mobile number: the user can enter the mobile number (say mobile-X) of the person to search for. In this case, the service will transform the user request to the following SPARQL:

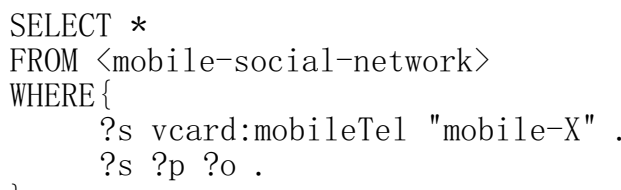

This query will be used to retrieve the profile of the person based on his/her mobile number. It should be noticed that the retrieved person profile contains the members he knows as they belongs to his/her profile. So that, the user can communicate with one of them. Moreover, if the mobile number belongs to a mobile company $y$ while the mobile number of the user requesting this service belongs to a mobile company $\mathrm{x}$, then a request is sent from $\mathrm{x}$ to $\mathrm{y}$.

- Searching based on a car number: the user can enter the car number (say car-X) of the person to search about. In this case, the service will transform the user request to the following SPARQL:

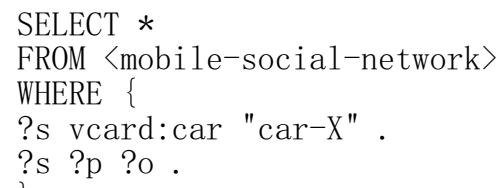

- Searching based on a person id number: similarly the user can retrieve the profile of a given person based on his/her id number. In this case, the service will transform the user request to the following SPARQL:

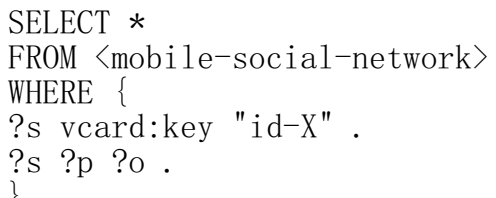

\section{Privacy Control For The Proposed SERVICE}

In the recent few years, some papers have investigated mobile social network related technologies, such as Dodgeball [17], [30]. However, to our knowledge, there is no paper having made an access right policy for friends and friends of a friend. In fact, most of them still depend on inherited access rights or customizable rights. One of the proposed methods in addressing such issue is to consider the mobile sharing as privacy-trust decision problem as suggested by [15], [19]. The approach takes into consideration the properties of a social relationship based on related data stored in a mobile device. The author binds the relation or strength of the social with the sensitivity of the resource to be shared at the initialization of the user profile. However, a privacy trust decision suggests that a friend can inherit his inviter access rights without any restrictions, which again raises the awareness of privacy concerns as well as the inherited access right dilemma.

Other solutions proposed for addressing privacy issues include distortion of information [7], context sensitive system adaptation [16], feedback loops [16], and mechanisms for access control [22]. However, most of these solutions typically involve a single aspect of privacy and does not decide what and how one might choose to disclose certain private information and to whom and to what extent. In addition to that, the issue of inherited access rights is still exists even though it can be adjusted later (i.e after the requester is added to the user friend list or to one of the trusted group) as illustrated in Figure 3.

Mobile social networking systems must give users sufficient control of their personal data, especially their personal information [13]. For instance, Twitter and Jaiku [Site 16] allow users to set their information private or public. However, all the user's posts will be either public or private. It would be better if there can be options that would let the user make some posts public and others private as well as deciding what to share and with whom. The traditional method in adding users/groups is portrayed in Figure 3. We can easily notice that the user initially can get access to the inviter profile unless he is added to one of the customized groups that has access rights to certain information. However, a better mechanism is needed that decides what users are allowed to view from the moment he is invited and how the whole user social network can be managed and organized. We urge that a person (in mobile social networking) can decide whom to invite via SMS. Prior to the invitation, the user can decide on what level of information he is willing to share with the new potential friend. For instance, the inviter may consider his location as sensitive for a friend who is not very closed to his social/relation, thus, the inviter can decide whether to disclose this info with the new friend or not yet.

Similarly, for his photo albums or address book. Moreover, the inviter can decide on whether the new friend can inherit the inviter rights to new friends, also he can decide if the new friend (friend of a friend) is allowed to publish the inviter's location to the invitee friends and friends of friends, etc. Figure 4 illustrates the logical sequence steps involved with the proposed method.

The proposed policy has the following structure (Figure 5) and vocabulary (Figure 6 and 7).

Where access indicates whether the user can access his inviter shared folders. Share is a feature that allows users to share the inviter info with their friends and friend of friends and invite is another feature that restricts users from inviting other users to the inviter social network. For instance, the following policy says that ahmad who has the uid=112 invites the user who has uid=111 with the following access rights. He is granted a limited access to the folder amman_gallary which indicates that the user 111 can only view the inviter's amman_gallary folder and not his friends. Moreover, he can only invited the inviter's (uid=112) friends to his social network. 
PAPER

Connecting Mobile Users Through Mobile Social Networks

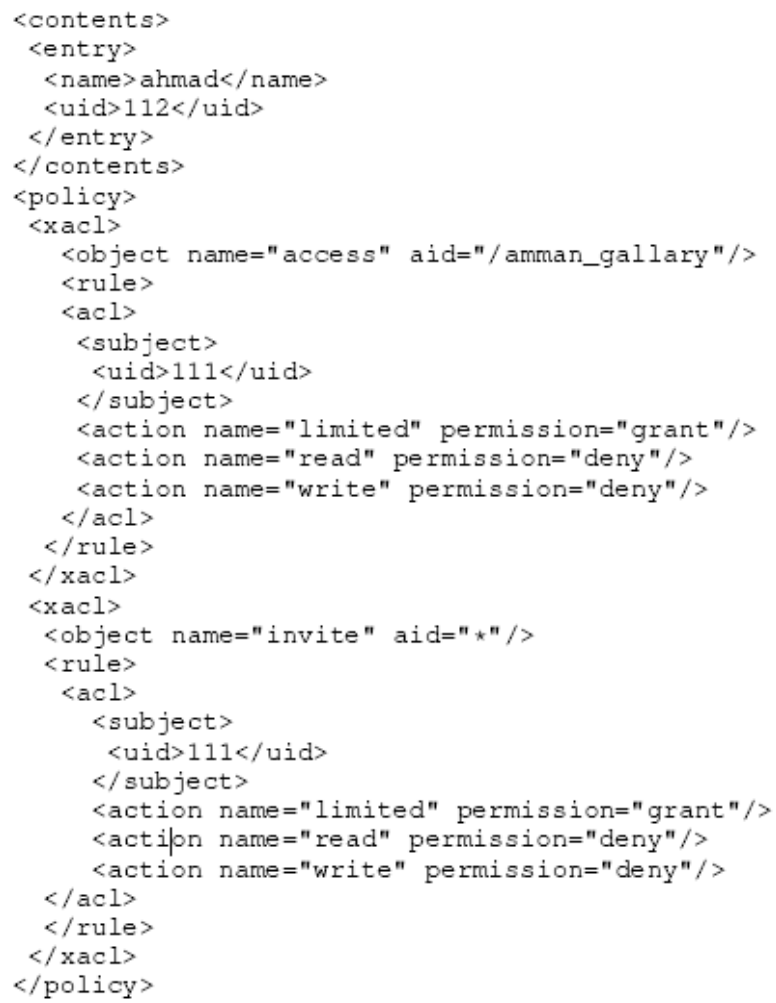

\section{RELATED WORK}

Several mobile social networking services have been proposed in the literature. However, these services are either based on social web sites or can be used to retrieve location of persons whose profiles match some attributes. In contrast to these services, our proposed service will create a social network for mobile users. The created social network, which is resided on the mobile phone company server, will be used as a base for other subservies.

Chang et. al. [9] proposed an interactive location-based system that supports social services. The system can be used to locate persons whose profiles match some attributes requested by the service.

A wireless virtual social network called PeopleNet is proposed in [23]. Using PeopleNet, a user can exchange information with other users via mobile phones.

Meet Now Live [Site 11] is a mobile social networking service that can be used by barflies and club-hoppers to transmit their real-time location in and around New York City.

Sniff [Site 12] (Social Network Integrated Friend Finder) is a mobile application that enables users to locate contacts in real-time via SMS or on Facebook, providing users with a detailed map view of their friends' current locations. However, users should provide the permission to the service to allow locating them by their friends.
Trapster [Site 13] is a free service that uses mobile phones and other types of devices to allow users to share the location of police speed traps.

Whrrl [Site 14] is a mobile social discovery service designed for BlackBerry device platform. It provides users with features like local discovery, mapping and microblogging via the addition of business components like events and calendar features. "Whrrl catalogs each user's history of places visited and events attended, mapping their behavior with color-coded icons that illustrate whether the experience was positive, neutral or negative. Users may share ratings and reviews as well as instantly update their location or status within their circle of contacts, be they business or casual".

Xumii (Site 8) is a service that can be used to call and message friends across social networks, create and join public and private groups across communities, update the user's status on all his/her identities, and upload photos and snapshots to Facebook, MySpace and Flickr. Users can connect, using any standard mobile browser, to their favourite social networks. Also, they can simultaneously stay in touch with their friends on Facebook, MySpace, Twitter and Flickr, with no need to move from one website to another or one application to another.

\section{CONCLUSION}

Most of the social networking services are online services that emphasize on building and reflecting social relations among people. We have presented in this paper a mobile service for building a social network for linking mobile users. The constructed social network will be maintained and updated regularly in the server of the mobile company.

Though the main objective of the proposed service is to construct a social network, it consists of several subservices such as: group finding (a user can search the group members located in a given zone), instant message ( a user can send message to his/her group members), and other services. We used the RDF language for representing mobile user profiles and the SPARQL query language for searching the RDF data representing user profiles. It should be noticed that the service is not a replacement of the existing services that can be used to connect to webbased social networks but a complementary to these services. That is, users can use the service to connect to their social groups anywhere and anytime without the need to create an account on web-based social network services. Users regardless of their disabilities, for example deaf or blind persons, can benefit from the proposed service. Additionally, to our knowledge, there is no paper having made an access right policy for friends and friends of a friend. To this end, we proposed a fine-grain privacy policy for restricting or granting access to specific data on mobile social networks. 
PAPER

Connecting Mobile Users Through Mobile Social Networks

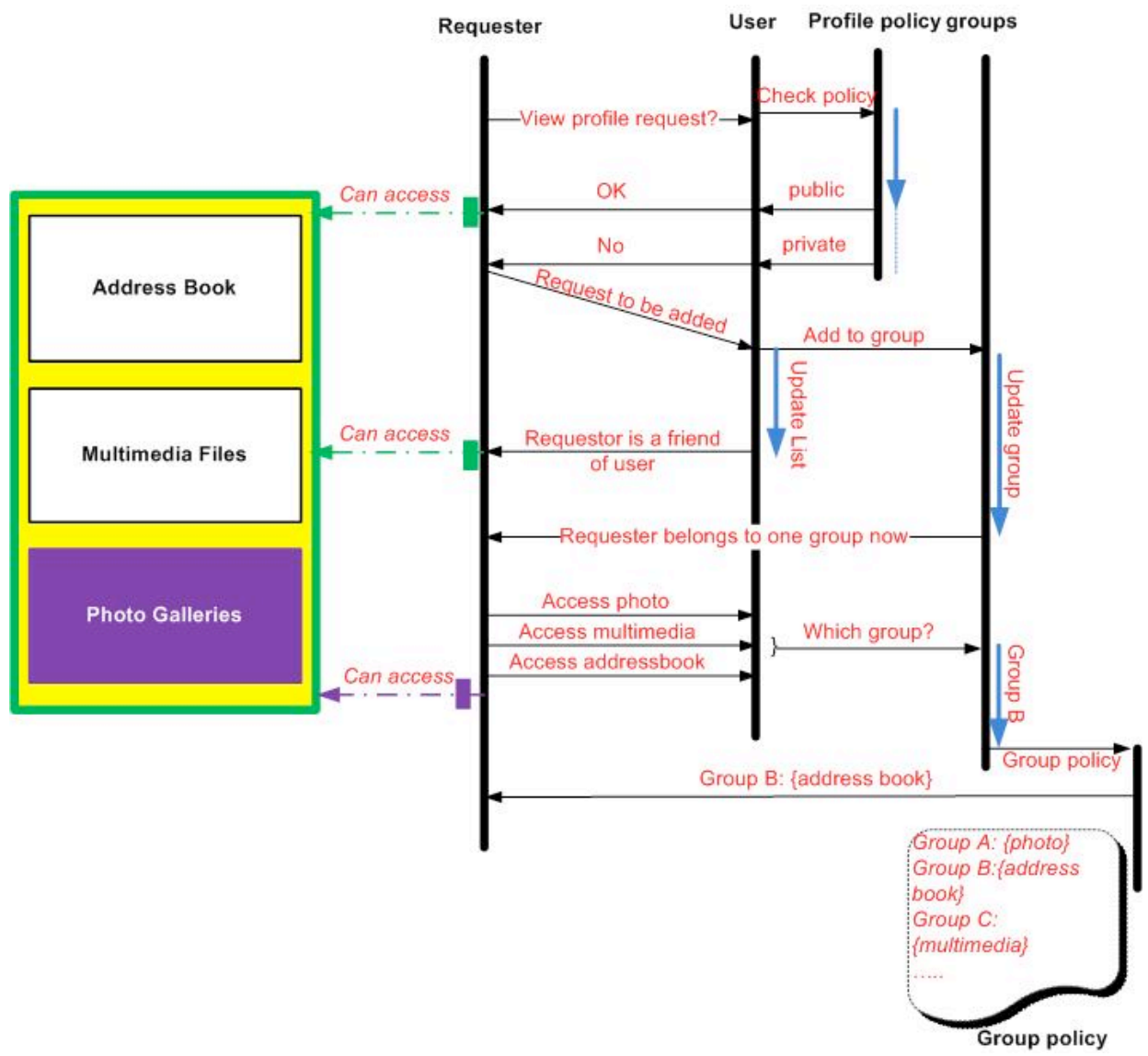

Figure 3. Traditional request (invite)/add access rights policy process.

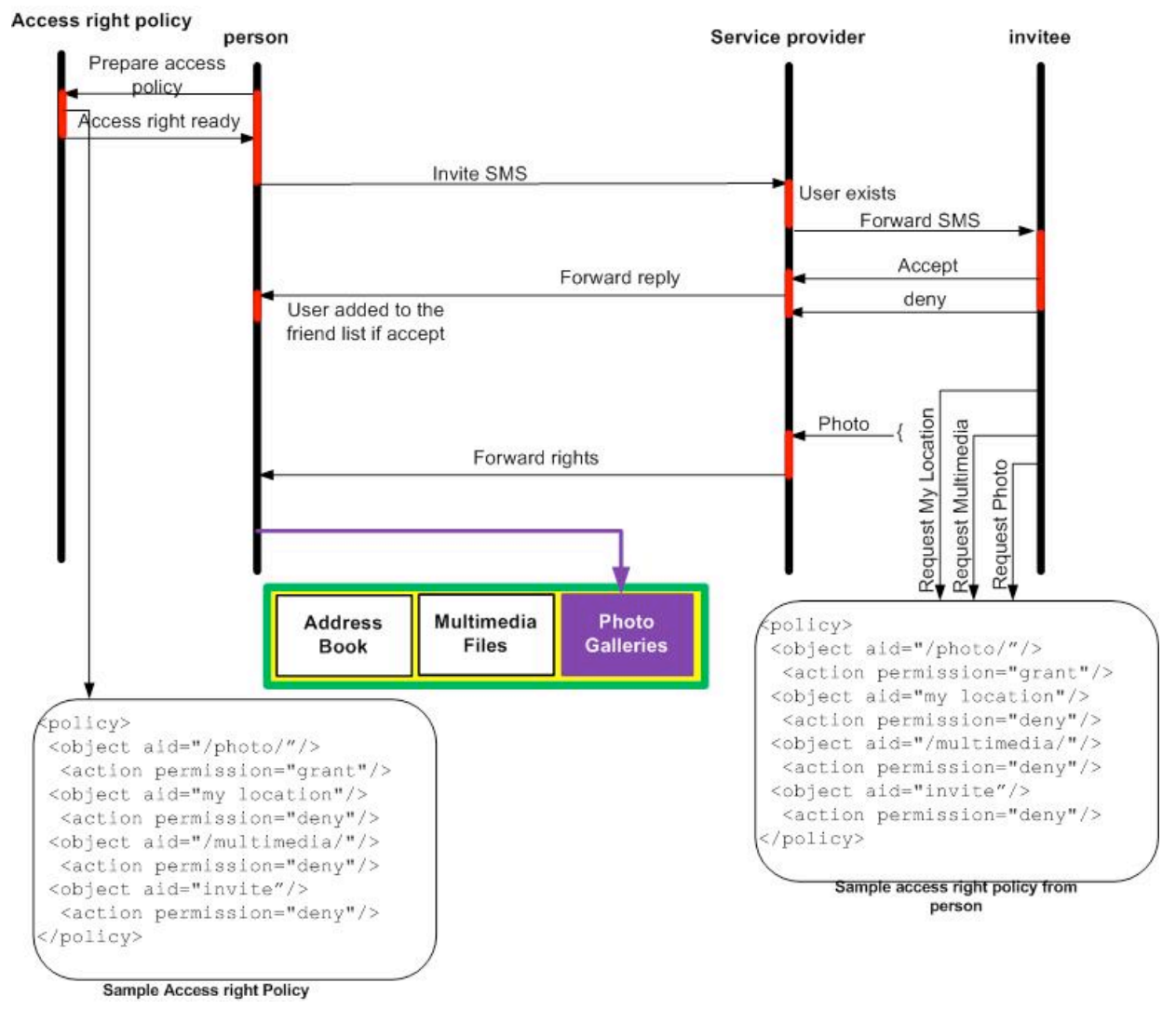

Figure 4. The proposed access rights policy process. 
PAPER

CONNecting Mobile Users Through Mobile Social Networks

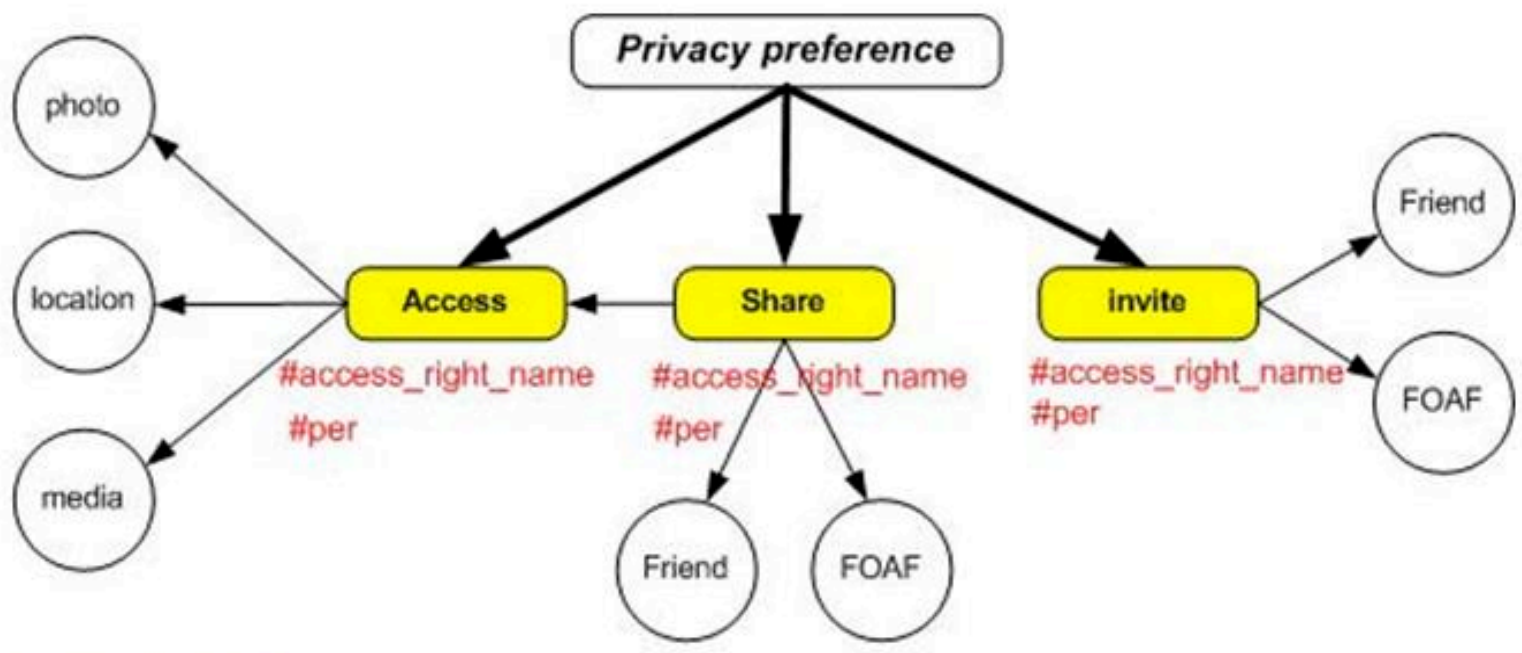

preference_name

\#access_right_name $=$ Read $/$ Write/ Limited

\#per $=$ Grant / Denied

Figure 5. Policy Structure.

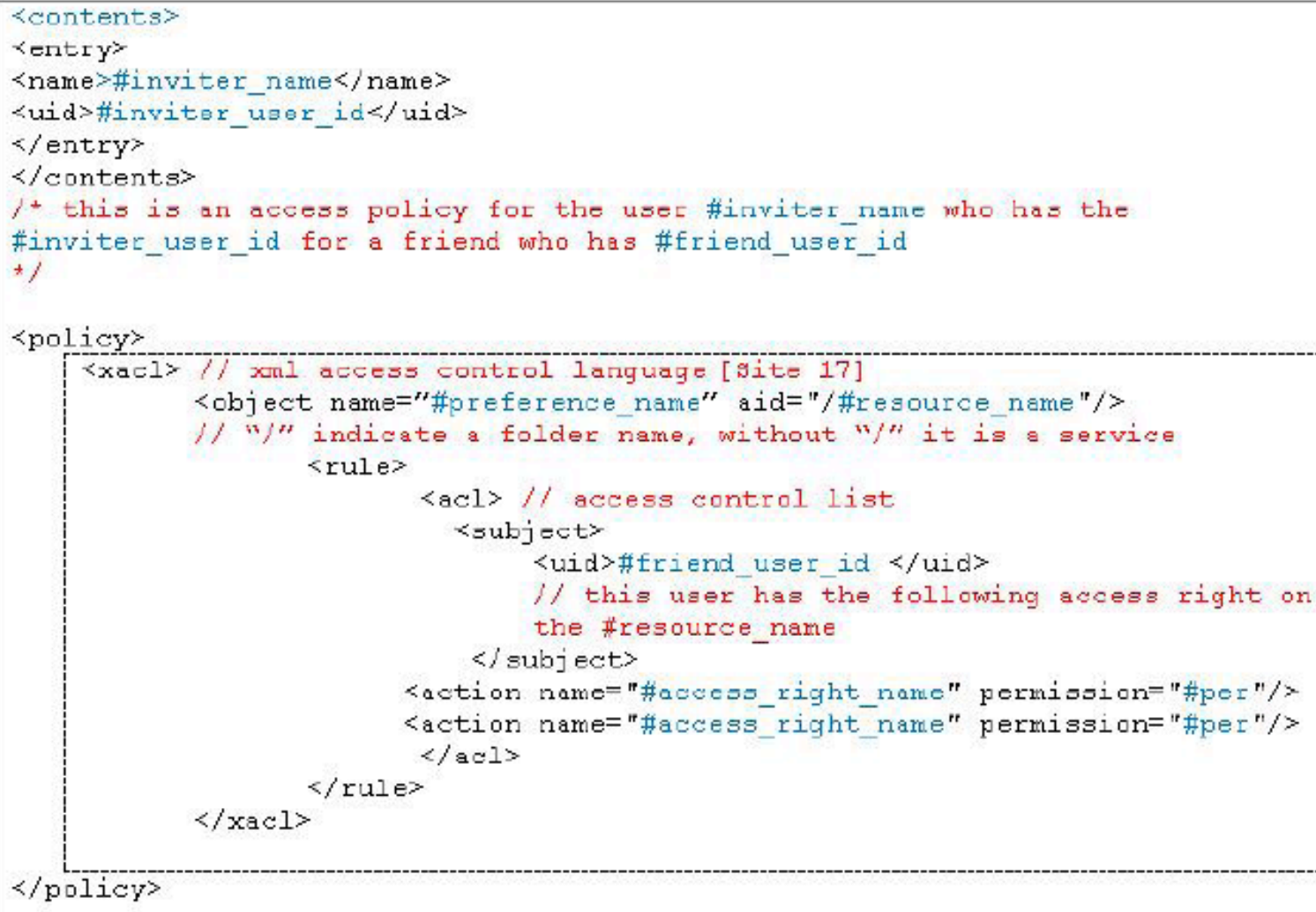

Figure 6. Policy Vocabulary.Conclusion 


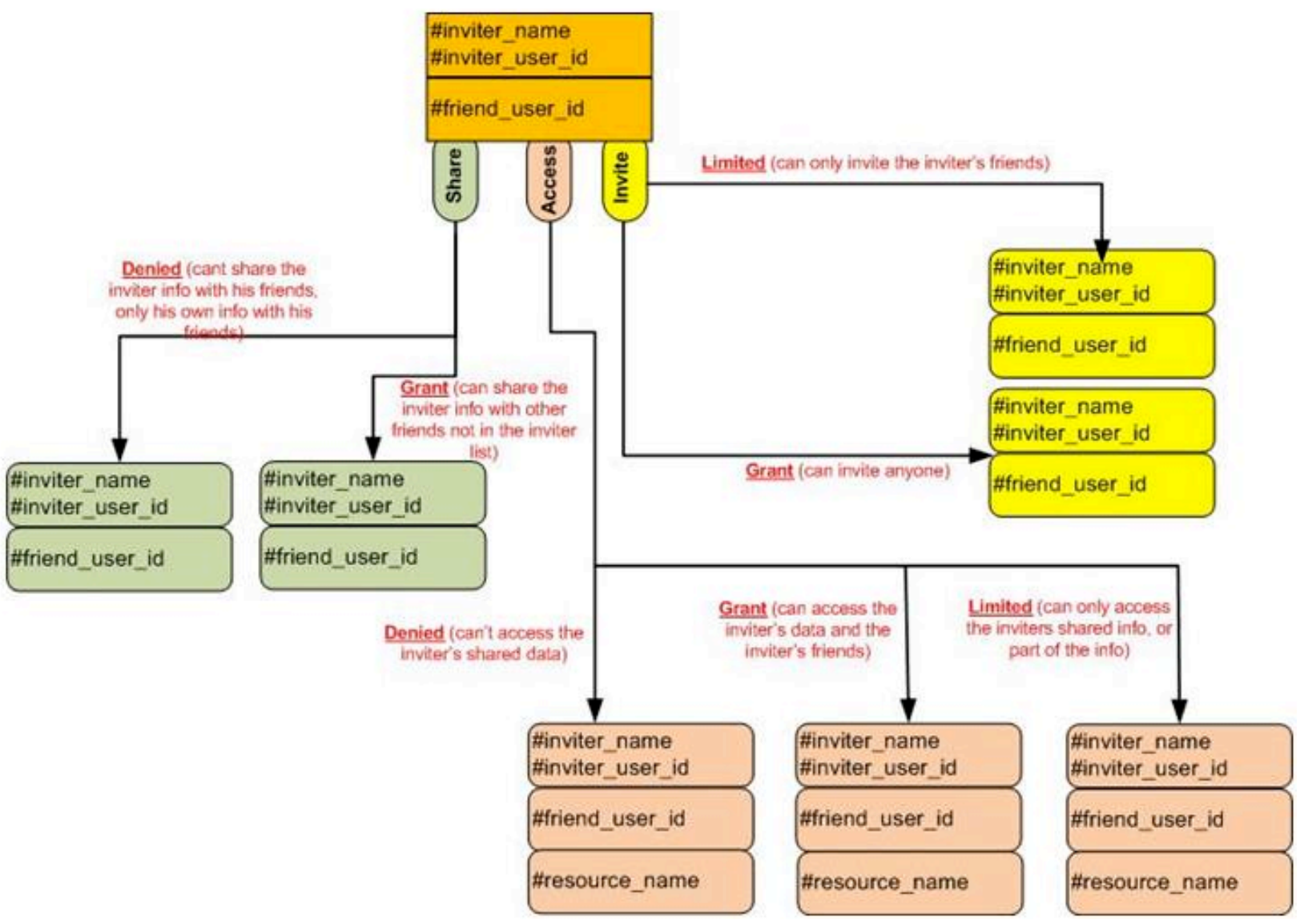

Figure 7. The use of the policy vocabulary.

\section{REFERENCES}

[1] Post-14 transitions - a survey of the social activity and social networking of blind and partially sighted young people. Technical report.

[2] Giuseppe Lugano . Understanding mobile relationships . In Human Centred Technology Workshop HCT , Pori, Finland, 2006.

[3] Faisal Alkhateeb, Jean-François Baget, and Jérôme Euzenat. Extending SPARQL with regular expression patterns (for querying RDF). Journal of web semantics, 7(2):57-73, 2009. http://dx.doi.org/10.1016/j.websem.2009.02.002

[4] Paolo Bellavista, Axel Küpper, and Sumi Helal. Locationbased services: Back to the future. IEEE Pervasive Computing, 7(2):8589, 2008. http://dx.doi.org/10.1109/MPRV.2008.34

[5] Danah Boyd and Nicole Ellison. Social network sites: Definition, history, and scholarship. Journal of Computer- Mediated Communication, 13(1), 2007. http://dx.doi.org/10.1111/j.1083-6101.2007. 00393.x

[6] Danah Boyd and Eszter Hargittai. Facebook privacy settings: Who cares? First Monday, 15(8), 2010.

[7] Michael Boyle, Christopher Edwards, and Saul Greenberg. The effects of filtered video on awareness and privacy. In CSCW 2000, pages 1-10. ACM Press, 2000. http://dx.doi.org/10.1145/ $\underline{358916.358935}$

[8] Dan Brickley and R.V. Guha. RDF vocabulary description language 1.0: RDF schema. Recommendation, W3C, 2004. http://www.w3.org/TR/rdf-schema/.

[9] Yao-Jen Chang, Hung-Huan Liu, Li-Der Chou, Yen-Wen Chen, and Haw-Yun Shin. A general architecture of mobile social network services. In International Conference on Convergence Information Technology, 2007, pages 151-156, nov. 2007.

[10] Ying Chen, Xiaoyan Chen, Xiaocheng Ding, Fangyan Rao, and Dong Liu. Bluelocator: Enabling enterprise locationbased serv- ices. Mobile Data Management, IEEE International Conference on, 0:167, 2002.

[11] Rui Cheng, Zhuo Yang, and Feng Xia. izone: A location based mobile social networking system. In Third International Symposium on Parallel Architectures, Algorithms and Programming (PAAP 2010), pages 33-38, December 2010.

[12] Rui Cheng, Zhuo Yang, and Feng Xia. A mobile-based service for building social networks for mobile users. In Third International Conference on Intelligent Systems, Modelling and Simulation (ISMS'2012), pages 545-549, February 2012.

[13] Nathan Eagle and Alex Pentland. Social serendipity: mobilizing social software. IEEE Pervasive Computing, 4(2):28-34, 2005. http://dx.doi.org/10.1109/MPRV.2005.37

[14] Nicole B. Ellison, Charles Steinfield, and Cliff Lampe. The benefits of facebook "friends": Exploring the relationship between college students' use of online social networks and social capital. Journal of Computer-Mediated Communication, 12(4), 2007. http://dx.doi.org/10.1111/j.1083-6101.2007.00367.x

[15] Lugano Giuseppe. Designing for societal development: Enhancing mobile social software through social capital. In International Social capital and networks of trust congress, 2007.

[16] Scott E. Hudson and Ian E. Smith. Techniques for addressing fundamental privacy and disruption tradeoffs in awareness support systems. In CSCW, pages 248-257, 1996.

[17] Ls Humphreys. Mobile social networks and social practice: A case study of dodgebal. Journal of Computer-Mediated Communication, 13(1), 2007. http://dx.doi.org/10.1111/j.1083-6101.2007. 00399.x

[18] Rich Ling. The Mobile Connection : The Cell Phone's Impact on Society (The Morgan Kaufmann Series in Interactive Technologies). Morgan Kaufmann, May 2004.

[19] Giuseppe Lugano. Mobile social software: Definition, scope and applications. In eChallenges 2007 Conference, pages 1434-1441, Netherlands, 2007. 
[20] Giuseppe Lugano and Pertti Saariluoma. To share or not to share: Supporting the user decision in mobile social software applications. In User Modeling, pages 440-444, 2007.

[21] Frank Manola and Eric Miller. RDF primer. Recommendation, W3C, 2004. http://www.w3.org/TR/rdf-primer/.

[22] Gloria Mark, Ludwin Fuchs, and Markus Sohlenkamp. Supporting groupware conventions through contextual awareness. In ECSCW, pages 253-, 1997.

[23] Mehul Motani and Vikram Srinivasan. Peoplenet: engineering a wireless virtual social network. In in Proceedings of ACM MobiCom, pages 243-257, 2005.

[24] Eric Prud'hommeaux and Andy Seaborne. SPARQL query language for RDF. Recommendation, W3C, January 2008. http://www.w3.org/TR/rdf-sparql-query/.

[25] A.R. Radcliffe-Brown. On social structure. Journal of the Royal Anthropological Institute, 70:1-12, 1940.

[26] Bharat Rao and Louis Minakakis. Evolution of mobile locationbased services. Commun. ACM, 46(12):61-65, December 2003. http://dx.doi.org/10.1145/953460.953490

[27] Jochen H. Schiller and Agnès Voisard, editors. Location-Based Services. Morgan Kaufmann, 2004.

[28] Stefan Steiniger, Moritz Neun, and Alistair Edwardes. Foundations of location based services. Cartouche - lecture notes on lbs, v. $1.0,2006$. http://www.geo.unizh.ch/publications/cartouche/lbs lecturenotes steinigeretal2006.pdf.

[29] Barry Wellman and S.D. Berkowitz. Social structures: A network approach. Cambridge: Cambridge University Press, 1988.

[30] Nina D. Ziv and Bala Mulloth. An exploration on mobile social networking: Dodgeball as a case in point. In ICMB, page 21, 2006.

[31] Faisal Alkhateeb. "A Mobile-Based Service for Building Social Networks for Mobile users". In Proceeding of the 3rd IEEE International Conference on Intelligent Systems, Modelling and Simulation (ISMS'12), ISBN: 978-0-7695-4668-1, pp.545-549, Kota Kinabalu, Malaysia, 8 - 10 February 2012.

\section{WEBSITES LIST}

Site 1: Social networking service, last visited October 20, 2011. (Wikipedia): http://en.wikipedia.org/wiki/Social $\backslash n$ etworking \service

Site 2: Facebook: http://www.facebook.com/

Site 3:Twitter: http://twitter.com/

Site 4:LinkedIn: www.linkedin.com/

Site 5: MySpace: www.myspace.com/

Site 6: Flickr: www.flickr.com/

Site 7: Hi5 : http://hi5.com/

Site 8: Xumii : http://www.myriadgroup.com/MobileOperators/Xumii $\ \% 20$ Social $\ \% 20$ Networking.aspx and http://xumii.com/v2/index.jsp

Site 9: Foaf vocabulary: $x$ mlns.com/foaf/0.1/

Site 10: Vcard vocabulary :www.w3.org/TR/vcard-rdf/

Site 11: Meet-now-live http://www.meetnowlive.com/

Site 12: sniff : https://www.sniffu.com/

Site 13: Trapster : trapster.com/

Site 14: Whrrl : http://www.whrrl.com/
Site 15: Google+ : https://plus.google.com/

Site 16: Jaiku : http://jaiku.com/

\section{AUTHORS}

Faisal Alkhateeb is an assistant professor in the department of computer science at Yarmouk University. He holds a B.Sc. from Yarmouk University, 1999; a M.Sc. from Yarmouk University, 2003; a M.Sc. from Grenoble 1, 2004; and Ph.D. from Grenoble 1, 2008. He is interested in knowledge-based systems, knowledge representation and reasoning, constraint satisfaction and optimization problems, intelligent systems, Semantic Web, and Artificial Intelligence.

Ahmed M. Manasrah is a senior lecturer at the Faculty of Information Technology and Computer Sciences, Yarmouk University, Irbid, Jordan since 2010. Dr. Manasra served as the Deputy Director (Research and Innovation) and the Head of iNetmon project at the National Advanced IPv6 Centre (NAv6) in Universiti Sains Malaysia. Since 2009. Dr. Manasra started his career as a web developer at Telaterra LLC from 2003 until 2004. Between 2005 until 2008, Dr. Ahmed worked as senior research officer in NRG, Universiti Sains Malaysia. He is interested in network monitoring and security.

Iyad Abu Doush is an Assistant Professor in the Department of Computer Science at Yarmouk University, Jordan. Dr. Abu Doush has received his BSc. in Computer Science from Yarmouk University, Jordan, 2001, and his M.S. from Yarmouk University, Jordan, 2003. He earned his Ph.D. from the Computer Science Department at New Mexico State University, USA, 2009. Since then, he has been a professor of computer science, Yarmouk University, Jordan. He has published more than 20 articles in international journals and conferences. His research interests include assistive technology, intelligent interfaces, and multimodal interfaces. Other research interests include human computer interaction, computational intelligence, and collaborative virtual environments.

Eslam Al Maghayreh is an Assistant Professor in the Department of Computer Science at Yarmouk University (Jordan) since June 2008. He has received his PhD degree in Computer Science from Concordia University (Canada) in 2008, his M.Sc. degree in Computer Science from Yarmouk University in 2003, and his B.Sc. degree in Computer Science from Yarmouk University in 2001. His research interests include: Distributed systems, multiagent systems, and runtime verification of distributed programs.

Manuscript received 02 August 2012. Published as resubmitted by the authors 23 September 2012. 